\title{
The Probabilistic and Reliable Connected Power Dominating Set Problems
}

\author{
Ou Sun · Neng Fan
}

August 28, 2018

\begin{abstract}
As a variation of minimum dominating set problem, the power dominating set problem is proposed to achieve the complete observation of a power system by placing the smallest number of PMUs. Under different contingencies consisting line outages or PMU losses, the reliability of observation for each bus should be guaranteed to ensure the security and robustness of the power system. Thus, the probabilistic power dominating set (PPDS) problem is proposed in this paper. Given a pre-specified reliability level for each bus in the power system to be observed and known distribution of random events, an integer programming formulation, with consideration of zero-injection property, is presented for the PPDS problem. Additionally, the reliable connected power dominating set problem is studied to meet two requirements, including the connectivity of the PMU subgraph and the reliability of the connectivity of this subgraph. Numerical experiments based on several IEEE test cases are performed to find the best deployment of PMUs satisfying different requirements.
\end{abstract}

Keywords Power dominating set problem · Probabilistic - Zero-Injection Buses · Integer Programming $\cdot$ Reliability $\cdot$ Connectivity

\section{Introduction}

In order to continuously monitor the health of a power system, some measurement devices are usually incorporated into the system. The phasor measurement unit (PMU) is such a frequently-used device which can collect voltage phasor for each bus and current phasor for each transmission line in the power system. By synchronized measurement, PMUs can help to gain complete observability of a power system. Due to the high expense of PMUs, the smallest number of them is needed to place on some buses without the loss of complete observation of the system. Following such

O. Sun · N. Fan $(\bowtie)$

Department of Systems and Industrial Engineering, University of Arizona, Tucson, AZ 85721, USA.

E-mail: nfan@email.arizona.edu 
idea, the power dominating set (PDS) problem is proposed in [1], in which the PDS problem is proved to be a NP-hard problem.

Given a set of elements $\mathscr{U}=\{1,2, \cdots, n\}$ (universe) and a family $\mathscr{F}$ of $m$ subsets $S_{1}, \cdots, S_{m}$, the set covering problem (SCP) is to identify the smallest number of sets whose union contains all elements in the universe. For a given graph $G=(V, E)$, a dominating set is a subset of $V$ such that every node not in the subset is connected to at least one member of the subset by some edge. The minimum dominating set (MDS) problem is to find a dominating set with smallest cardinality. Thus, the MDS problem can be considered as a special case of $\operatorname{SCP}\left(\mathscr{U}=V, \mathscr{F}=\left\{N\left[v_{i}\right]: v_{i} \in V\right\}\right.$, where $N\left[v_{i}\right]$ is the closed neighborhood of vertex $v_{i}$ ), and it is also a NP-hard problem [2].

The PDS problem has a close relationship to MDS. A power system graph (PSG) is usually described by an undirected graph $G=(V, E)$, where $V$ represents a set of buses $\left(v_{i} \in V\right)$ and $E$ represents a set of transmission lines. A subset $V_{Z} \subset V$ is usually used to describe a special set of buses, which are called zero-injection buses. They are those buses without generation or loading, and have some particular property, called zero-injection bus property, as explained below. In the PDS problem, PMUs are deployed to place on some buses (obtaining a subset $D \subseteq V$ ) with the goal of observing the whole power system by smallest number of PMUs. Two following physical laws need to be included when considering PDS: (1) Ohm's law: if bus $v_{i} \in D$, then $v_{i}$ and all its neighbors are all covered; (2) Kirchhoff's current law (zero-injection bus property): if $v_{i} \in V_{Z}$, and $v_{i}$ and all its neighbors (within closed neighborhood $N\left[v_{i}\right]$ ) except one are covered, then the only uncovered bus will be covered automatically. If only Ohm's law is considered, the PDS problem is equivalent to the MDS problem. However, if both properties are considered, the necessary number of PMUs can be reduced as a benefit of applying the zero-injection bus property. Due to large amount of investments to PMU technology, the PDS problem and its variations are widely studied recently, including multistage PMU placement problem [3,4], and multichannel PMU placement problem [5].

As studied in the literature for the SCP, the coverage relationship is not always deterministic, and thus the probabilistic set covering problem was proposed (see [6]). As a special case of the SCP, the MDS problem may suffer from uncertainty, which leads to the study of probabilistic MDS (PMDS) problem [7, 8].

In practice, it is not guaranteed that the installed PMUs and the transmission lines always function well. The contingencies consisting of line outages or PMU losses happen independently or simultaneously from time to time in practical power system. Under such cases, the goal becomes achieving a robust placement scheme under contingencies. The integer programming (IP) approaches are widely used when incorporating different contingency situations [3,9-16]. Most of them try to improve the reliability of the system by adding some redundant observability for each bus either in the constraints $[3,11-14]$ or in the objective $[9,10]$. However, either they do not consider PMU losses [3,11], or ignore the zero-injection bus property [12] or complete observability [13]. Considering the reliability level of PMUs and transmission lines are different from each other, the vulnerabilities of different parts of the power system are not the same. Thus, adding same amount of redundancy may be not enough or not necessary. Considering such issue, a robust and secure PMU placement will have further benefits. In $[15,16]$, the robustness is achieved by predefined 
probability of observability for each bus, in case of failures in the system. However, both of them omit the zero-injection bus property.

In this paper, with the consideration of potential failures on transmission lines and/or PMUs, we consider two types of reliability issues for PMU placements. The first one is called the probabilistic power dominating set (PPDS) problem, which ensures that each bus has a reliability level $1-\varepsilon_{i}$ to be observed in case of any failures $(\varepsilon \in(0,1))$. The second one is called the reliable connected power dominating set (RCPDS) problem, which extends the connected power dominating set (CPDS) problem, and requires the connectivity of PMU subgraph and reliability of the connectivity of this subgraph. The CPDS problem was proposed in [5], and the corresponding PMU placements through the CPDS can reduce the cost of processing stations and communication infrastructure related to collecting data from PMUs. In both proposed problems, the zero-injection bus property will be included. To efficiently solve them, novel reformulation techniques will be studied and new algorithms will be designed.

The remainder of this paper is organized as follows. The PPDS problem with consideration of zero-injection bus property is formulated, and also reformulated as a mixed integer program (MIP) in Section 2. In Section 3, we study the CPDS problem with the reliability requirements in case of failures. In Section 4, numerical experiments on several IEEE test cases are performed and compared for different models. Section 5 concludes the paper.

\section{The Probabilistic Power Dominating Set Problem}

2.1 Probabilistic power dominating set problem

Given a PSG $G=(V, E)$, we have the following information:

- $A=\left(a_{i j}\right)_{|V| \times|V|}$ : a neighborhood matrix, where $a_{i j}=1$ if $i=j$ or bus $v_{i}$ and $v_{j}$ are connected by a transmission line, and $a_{i j}=0$ otherwise.

- $N\left[v_{i}\right]$ : closed neighborhood of bus $v_{i}$, including bus $v_{i}$ and its neighbors linked by some transmission lines, whereas $N\left(v_{i}\right)$ denotes the open neighborhood for bus $v_{i}$ which only includes the neighbors of bus $v_{i}$.

- $Z_{i}$ : zero-injection bus, where $Z_{i}=1$ if bus $v_{i}$ is a zero-injection bus, and $Z_{i}=0$ otherwise. The notation $V_{Z} \subset V$ denotes the set of all the zero-injection buses.

The decision variables for the PDS problem include

$-x_{i}: x_{i}=1$ if a PMU is placed on bus $v_{i}$, and $x_{i}=0$ otherwise.

- $y_{i j}: y_{i j}=1$ if bus $v_{i}$ is a zero-injection bus and it can provide a one time coverage for bus $v_{j}$ because of the zero-injection bus property, and $y_{i j}=0$ otherwise.

In the deterministic version of the PDS problem, the following IP formulation proposed in [17] is applied to ensure that each bus in the power system is observed at 
Fig. 1 PMU Placement on IEEE 14-Bus System

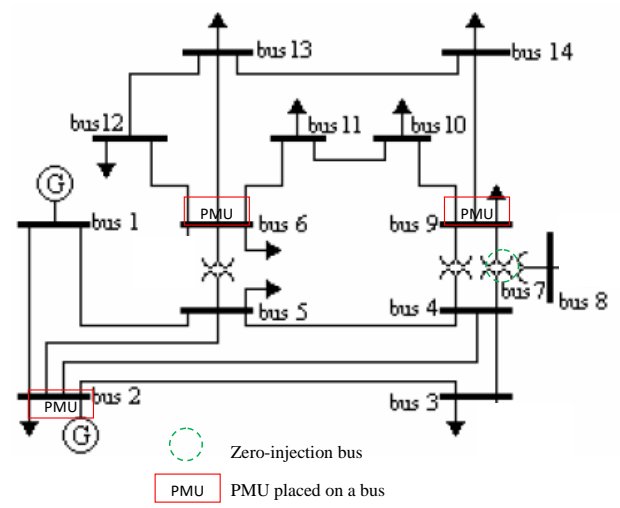

least once:

[PDS

$$
\begin{array}{ll}
\min _{x, y} & \sum_{i: v_{i} \in V} x_{i} \\
\text { s.t. } & \sum_{j: v_{j} \in N\left[v_{i}\right]} x_{j}+\sum_{j: v_{j} \in N\left[v_{i}\right]} Z_{j} y_{j i} \geq 1, \forall v_{i} \in V \\
& \sum_{j: v_{j} \in N\left[v_{i}\right]} y_{i j} \leq Z_{i}, \forall v_{i} \in V \\
& y_{i j}=0, \forall v_{i} \notin V_{Z} \\
& x_{i}, y_{i j} \in\{0,1\}, \forall v_{i}, v_{j} \in V
\end{array}
$$

where the objective (1a) is to minimize the number of PMUs. Constraints (1c)-(1d) limit that each zero-injection bus can contribute at most one time of bus observation within the neighborhood $N\left[v_{i}\right]$. The left-hand-side of (1b) measures the times of observation for bus $v_{i}$. Comparing with the MDS, the first term $\sum_{j: v_{j} \in N\left[v_{i}\right]} x_{j}$ appears in both problems. However, because of the existence of zero-injection bus property, the additional term $\sum_{j: v_{j} \in N\left[v_{i}\right]} Z_{j} y_{j i}$ counts the possible observations.

To better illustrate the problem, we use the IEEE 14-Bus system as an example (see Fig. 1). There are 14 buses in the system, where bus 7 is a zero-injection bus. The minimum amount of PMUs needed to ensure the complete bus observability is 3 , and these PMUs can be on bus 2, bus 6, and bus 9. If a PMU is placed on bus 6, bus 6 is observed directly, and buses 5,11,12,13 are observed by Ohm's law. The same effects can be achieved with the placement of PMUs on bus 2 and bus 9. By Ohm's law, all the buses except for bus 8 can be observed. By applying Kirchhoff's current law on bus 7 (within $N\left[v_{7}\right]$, buses 4, 7, 9 are already observed), bus 8 is also observed. Thus, 3 PMUs in total can guarantee the full observation of the system.

If there are random failures from transmission lines or PMUs, to achieve a reliable coverage for each bus, we propose the following probabilistic power dominating set (PPDS) problem: finding a smallest subset of $V$ in the PSG $G=(V, E)$ such that each bus has a reliability level $1-\varepsilon_{i}$ to be observed in case of any failures, where 
$\varepsilon_{i} \in(0,1)$. To formulate this new requirement, the constraints (1b) can be modified to the following chance constraints

$$
\mathbb{P}\left(\sum_{j: v_{j} \in N\left[v_{i}\right]} x_{j}+\sum_{j: v_{j} \in N\left[v_{i}\right]} Z_{j} y_{j i} \geq 1\right) \geq 1-\varepsilon_{i}, \forall v_{i} \in V .
$$

A chance constraint is difficult to deal with, and the main reason comes from the non-convexity of the feasible regions with general probability distributions. However, in some cases, it can be convex relaxed [18] or linearized [19].

\subsection{Random failures of transmission lines}

If the transmission lines suffer from some randomly occurred failures and have some probabilities to fail, the PDS problem will not be deterministic any more. Under such situation, the variable $a_{i j}$ becomes a random variable $\widetilde{a}_{i j}$ following some distribution. In this paper, we assume that the transmission line encounters a random failure with probability $1-p_{i j}$, and survives with probability $p_{i j}$. That is the random variable $\widetilde{a}_{i j}$ follows Bernoulli distribution with parameter $\mathbb{P}\left(\widetilde{a}_{i j}=1\right)=p_{i j}\left(0<p_{i j}<1\right)$ and $\mathbb{P}\left(\widetilde{a}_{i j}=0\right)=1-p_{i j}$, i.e., $\widetilde{a}_{i j} \sim \operatorname{Bernoulli}\left(p_{i j}\right)$. In addition, we assume that random variables $\widetilde{a}_{i j}$ 's are independent of each other.

With the above assumptions, we have Theorem 1 showing the reformulation of chance constraint (2).

Theorem 1 With random failures of transmission lines, the chance constraints (2) for the PPDS problem can be reformulated as

$$
x_{i}+Z_{i} y_{i i}+\sum_{j \in N\left(v_{i}\right)}\left(x_{j}+Z_{j} y_{j i}-x_{j} Z_{j} y_{j i}\right) \frac{\log \left(1-p_{i j}\right)}{\log \varepsilon_{i}} \geq 1, \text { for all } v_{i} \in V .
$$

Proof. Recall the definition of entry $a_{i j}$ in the matrix $A$ that $a_{i j}=1$ when $i=j$ or bus $v_{i}$ and $v_{j}$ are neighbors. Then, the chance constraint $\mathbb{P}\left(\sum_{j: v_{j} \in N\left[v_{i}\right]} x_{j}+\sum_{j: v_{j} \in N\left[v_{i}\right]} Z_{j} y_{j i} \geq\right.$ 1) $\geq 1-\varepsilon_{i}, \forall v_{i} \in V$ becomes

$$
\mathbb{P}\left(x_{i}+\sum_{v_{j} \in N\left(v_{i}\right)} \tilde{a}_{i j} x_{j}+Z_{i} y_{i i}+\sum_{v_{j} \in N\left(v_{i}\right)} \tilde{a}_{i j} Z_{j} y_{j i} \geq 1\right) \geq 1-\varepsilon_{i}, \forall v_{i} \in V .
$$

Since $x_{i}$ is a decision variable in this problem and it is not determined now, there are two cases to express the constraint considering different values of $x_{i}$ in the solution. Define a subset $D \subseteq V$ consists all of the nodes $v_{i} \in V$ where $x_{i}=1$ in the solution.

- If $v_{i} \in D, x_{i}=1$, constraint (4) is $\mathbb{P}\left(1+\sum_{j \in N\left(v_{i}\right)} \widetilde{a}_{i j} x_{j}+Z_{i} y_{i i}+\sum_{j \in N\left(v_{i}\right)} \widetilde{a}_{i j} Z_{j} y_{j i} \geq\right.$ 1) $\geq 1-\varepsilon_{i}, \forall v_{i} \in D$, which is always satisfied.

- If $v_{i} \notin D, x_{i}=0$, constraint (4) becomes

$$
\mathbb{P}\left(\sum_{j \in N\left(v_{i}\right)} \widetilde{a}_{i j} x_{j}+\sum_{j \in N\left(v_{i}\right)} \widetilde{a}_{i j} Z_{j} y_{j i}+Z_{i} y_{i i} \geq 1\right) \geq 1-\varepsilon_{i}, \forall v_{i} \in V \backslash D .
$$

For different values of term $Z_{i} y_{i i}$ in the solution, the above inequality can also be classified into two cases, 
- If $Z_{i} y_{i i}=1, \mathbb{P}\left(\sum_{j \in N\left(v_{i}\right)} \widetilde{a}_{i j} x_{j}+\sum_{j \in N\left(v_{i}\right)} \widetilde{a}_{i j} Z_{j} y_{j i}+1 \geq 1\right) \geq 1-\varepsilon_{i}$ is satisfied;

- If $Z_{i} y_{i i}=0$, constraint (5) is equivalent to

$$
\begin{aligned}
& \mathbb{P}\left(\sum_{j \in N\left(v_{i}\right)} \widetilde{a}_{i j} x_{j}+\sum_{j \in N\left(v_{i}\right)} \widetilde{a}_{i j} Z_{j} y_{j i} \geq 1\right) \geq 1-\varepsilon_{i}, \forall v_{i} \in V \backslash D \\
& \Leftrightarrow \mathbb{P}\left(\sum_{j \in N\left(v_{i}\right)} \widetilde{a}_{i j} x_{j}+\sum_{j \in N\left(v_{i}\right)} \widetilde{a}_{i j} Z_{j} y_{j i}=0\right) \leq \varepsilon_{i}, \forall v_{i} \in V \backslash D \\
& \Leftrightarrow \mathbb{P}\left(\sum_{j \in N\left(v_{i}\right)} \widetilde{a}_{i j}\left(x_{j}+Z_{j} y_{j i}\right)=0\right) \leq \varepsilon, \forall v_{i} \in V \backslash D \\
& \Leftrightarrow \Pi_{j \in N\left(v_{i}\right)} \mathbb{P}\left(\widetilde{a}_{i j}\left(x_{j}+Z_{j} y_{j i}\right)=0\right) \leq \varepsilon_{i}, \forall v_{i} \in V \backslash D \\
& \Leftrightarrow \Pi_{j \in N\left(v_{i}\right)}\left(1-p_{i j}\right)^{x_{j}+Z_{j} y_{j i}-x_{j} Z_{j} y_{j i}} \leq \varepsilon_{i}, \forall v_{i} \in V \backslash D \\
& \Leftrightarrow \sum_{j \in N\left(v_{i}\right)}\left(x_{j}+Z_{j} y_{j i}-x_{j} Z_{j} y_{j i}\right) \log \left(1-p_{i j}\right) \leq \log \varepsilon_{i}, \forall v_{i} \in V \backslash D \\
& \Leftrightarrow \sum_{j \in N\left(v_{i}\right)}\left(x_{j}+Z_{j} y_{j i}-x_{j} Z_{j} y_{j i}\right) \frac{\log \left(1-p_{i j}\right)}{\log \varepsilon_{i}} \geq 1, \forall v_{i} \in V \backslash D .
\end{aligned}
$$

The inequality (6b) exists because the probability for event $\sum_{j \in N\left(v_{i}\right)} \widetilde{a}_{i j} x_{j}+$ $\widetilde{a}_{i j} Z_{j} y_{j i} \geq 1$ to be greater than or equal to $1-\varepsilon_{i}$ is equivalent to the probability of event $\sum_{j \in N\left(v_{i}\right)} \widetilde{a}_{i j} x_{j}+\widetilde{a}_{i j} Z_{j} y_{j i}=0$ to be less than or equal to $\varepsilon_{i}$. Inequality (6d) follows because the probability of the summation of $\widetilde{a}_{i j}\left(x_{j}+Z_{j} y_{j i}\right)$ 's to be 0 equals to probability of each $\widetilde{a}_{i j}\left(x_{j}+Z_{j} y_{j i}\right)$ to be 0 . And the probability of each $\widetilde{a}_{i j}\left(x_{j}+Z_{j} y_{j i}\right)$ to be 0 equals to the product of each probability of $\widetilde{a}_{i j}\left(x_{j}+Z_{j} y_{j i}\right)$ to be 0 because of the independence of each $\widetilde{a}_{i j}$. Inequality (6e) follows from the technique of calculating $\mathbb{P}\left(\widetilde{a}_{i j}\left(x_{j}+Z_{j} y_{j i}\right)=0\right)$. A similar technique was used in [19]. $\mathbb{P}\left(\widetilde{a}_{i j}=0\right)=1-p_{i j}$, and both terms $x_{j}$ and $Z_{j} y_{j i}$ can select value from $\{0,1\}$. Thus, the value of term $x_{j}+Z_{j} y_{j i}$ can be an element in the set $\{0,1,2\}$. When $x_{j}+Z_{j} y_{j i}=0, \mathbb{P}\left(\widetilde{a}_{i j}\left(x_{j}+Z_{j} y_{j i}\right)=0\right)=$ $1=\left(1-p_{i j}\right)^{0}$; when $x_{j}+Z_{j} y_{j i}=1, \mathbb{P}\left(\widetilde{a}_{i j}\left(x_{j}+Z_{j} y_{j i}\right)=0\right)=1-p_{i j}=(1-$ $\left.p_{i j}\right)^{1}=\left(1-p_{i j}\right)^{1+0-0 / 0+1-0}$; when $x_{j}+Z_{j} y_{j i}=2, \mathbb{P}\left(\widetilde{a}_{i j}\left(x_{j}+Z_{j} y_{j i}\right)=0\right)=$ $1-p_{i j}=\left(1-p_{i j}\right)^{1}=\left(1-p_{i j}\right)^{1+1-1}$. Generalize the above three cases, we can

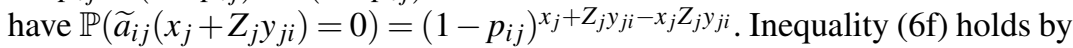
taking logarithm of both sides of the inequality. We can obtain $(6 \mathrm{~g})$ because $\varepsilon_{i} \in(0,1)$ and $\log \varepsilon_{i} \in(-\infty, 0)$.

Consider both cases of $Z_{i} y_{i i}=1$ and $Z_{i} y_{i i}=0$ when $v_{i} \notin D$, we can have

$$
Z_{i} y_{i i}+\sum_{j \in N\left(v_{i}\right)}\left(x_{j}+Z_{j} y_{j i}-x_{j} Z_{j} y_{j i}\right) \frac{\log \left(1-p_{i j}\right)}{\log \varepsilon_{i}} \geq 1, \forall v_{i} \in V \backslash D .
$$

If we both take situations of $v_{i} \in D$ and $v_{i} \notin D$ into account, we will get,

$$
x_{i}+Z_{i} y_{i i}+\sum_{j \in N\left(v_{i}\right)}\left(x_{j}+Z_{j} y_{j i}-Z_{j} x_{j} y_{j i}\right) \frac{\log \left(1-p_{i j}\right)}{\log \varepsilon_{i}} \geq 1, \forall v_{i} \in V .
$$

The nonlinear term $x_{j} y_{j i}$ in (3) can be easily linearized by introducing $w_{i j}=x_{j} y_{j i}$ with constraints $w_{i j} \leq x_{j}, w_{i j} \leq y_{j i}, w_{i j} \geq x_{j}+y_{j i}-1$ and $w_{i j} \geq 0$. 
With the objective (1a), constraints (1c), (1d), (1e) and the reformulated constraints (3), we have the MIP formulation for the PPDS problem.

\subsection{Random failures of both transmission lines and PMUs}

When there are random failures from both transmission lines and PMUs, we can still obtain the similar reformulation, which is presented in Theorem 2. For the random failure of PMUs, let $\widetilde{b_{i}} \in\{0,1\}$ denote the random variable to indicate the failed PMU installed on bus $v_{i}$. We also assume that $\widetilde{a}_{i j} \sim \operatorname{Bernoulli}\left(p_{i j}\right)\left(0<p_{i j}<1\right), \widetilde{b}_{i} \sim$ Bernoulli $\left(q_{i}\right)\left(0<q_{i}<1\right)$ and they are independent to each other.

Theorem 2 With random failures of transmission lines and PMUs, the chance constraint (2) in the PPDS problem can be reformulated as

$$
\begin{aligned}
& Z_{i} y_{i i}+\sum_{j \in N\left(v_{i}\right)} \frac{\log \left(1-p_{i j} q_{j}\right)}{\log \varepsilon_{i}} x_{j}\left(1-Z_{j} y_{j i}\right)+\sum_{j \in N\left(v_{i}\right)} \frac{\log \left(1-p_{i j}\right)}{\log \varepsilon_{i}} Z_{j} y_{j i} \\
+ & \frac{\log \left(1-q_{i}\right)}{\log \varepsilon_{i}} x_{i} \geq 1, \text { for all } v_{i} \in V .
\end{aligned}
$$

Proof. The chance constraints (2) can be expressed as follows,

$$
\mathbb{P}\left(\widetilde{b}_{i} x_{i}+\sum_{j \in N\left(v_{i}\right)} \widetilde{a}_{i j} \widetilde{b}_{j} x_{j}+Z_{i} y_{i i}+\sum_{j \in N\left(v_{i}\right)} \widetilde{a}_{i j} Z_{j} y_{j i} \geq 1\right) \geq 1-\varepsilon_{i}, \forall v_{i} \in V
$$

We have two following cases including $v_{i} \in D$ and $v_{i} \notin D$, because the value of $x_{i}$ is undetermined.

- $v_{i} \in D, x_{i}=1, \mathbb{P}\left(\widetilde{b}_{i}+\sum_{j \in N\left(v_{i}\right)} \widetilde{a}_{i j} \widetilde{b}_{j} x_{j}+Z_{i} y_{i i}+\sum_{j \in N\left(v_{i}\right)} \widetilde{a}_{i j} Z_{j} y_{j i} \geq 1\right) \geq 1-\varepsilon_{i}, \forall v_{i} \in$ $D$. For different values of $Z_{i} y_{i i}$ in this solution, it can be classified into another two cases including $Z_{i} y_{i i}=1$ and $Z_{i} y_{i i}=0$.

- $Z_{i} y_{i i}=1, x_{i}=1, \mathbb{P}\left(\widetilde{b}_{i}+\sum_{j \in N\left(v_{i}\right)} \widetilde{a}_{i j} \widetilde{b}_{j} x_{j}+1+\sum_{j \in N\left(v_{i}\right)} \widetilde{a}_{i j} Z_{j} y_{j i} \geq 1\right) \geq 1-\varepsilon_{i}, \forall v_{i} \in$ $D$ is satisfied for sure.

$-Z_{i} y_{i i}=0, x_{i}=1$

$$
\begin{aligned}
& \mathbb{P}\left(\widetilde{b}_{i}+\sum_{j \in N\left(v_{i}\right)} \widetilde{a}_{i j} \widetilde{b}_{j} x_{j}+\sum_{j \in N\left(v_{i}\right)} \widetilde{a}_{i j} Z_{j} y_{j i} \geq 1\right) \geq 1-\varepsilon_{i}, \forall v_{i} \in D \\
\Leftrightarrow & \mathbb{P}\left(\widetilde{b}_{i}=0, \widetilde{a}_{i j} \widetilde{b}_{j} x_{j}=0, \widetilde{a}_{i j} Z_{j} y_{j i}=0, \forall j \in N\left(v_{i}\right)\right) \leq \varepsilon_{i}, \forall v_{i} \in D \\
\Leftrightarrow & \left(1-q_{i}\right) \Pi_{j \in N\left(v_{i}\right)} \mathbb{P}\left(\widetilde{a}_{i j} \widetilde{b}_{j} x_{j}=0 \text { and } \widetilde{a}_{i j} Z_{j} y_{j i}=0\right) \leq \varepsilon_{i}, \forall v_{i} \in D \\
\Leftrightarrow & \left(1-q_{i}\right) \Pi_{j \in N\left(v_{i}\right)}\left(1-p_{i j} q_{j}\right)^{x_{j}\left(1-Z_{j} y_{j i}\right)}\left(1-p_{i j}\right)_{j} y_{j i} \leq \varepsilon_{i}, \forall v_{i} \in D \\
\Leftrightarrow & \sum_{j \in N\left(v_{i}\right)} \log \left(1-p_{i j} q_{j}\right) x_{j}\left(1-Z_{j} y_{j i}\right)+\sum_{j \in N\left(v_{i}\right)} \log \left(1-p_{i j}\right) Z_{j} y_{j i} \\
& +\log \left(1-q_{i}\right) \leq \log \varepsilon_{i}, \forall v_{i} \in D \\
\Leftrightarrow & \sum_{j \in N\left(v_{i}\right)} \frac{\log \left(1-p_{i j} q_{j}\right)}{\log \varepsilon_{i}} x_{j}\left(1-Z_{j} y_{j i}\right)+\sum_{j \in N\left(v_{i}\right)} \frac{\log \left(1-p_{i j}\right)}{\log \varepsilon_{i}} Z_{j} y_{j i} \\
& +\frac{\log \left(1-q_{i}\right)}{\log \varepsilon_{i}} \geq 1, \forall v_{i} \in D
\end{aligned}
$$


We can write down the chance constraint as (10a) when $x_{i}=1$ and $Z_{i} y_{i i}=$ 0 . Then, we can get $(10 \mathrm{~b})$ intuitively. The inequality $(10 \mathrm{c})$ comes from the independence of event $\widetilde{b}_{i}=0$ to other events. The inequality (10d) is true because of the following analysis. To simplify the clarification, $\forall v_{j} \in N\left(v_{i}\right)$, let $\mathbb{P}\left(\widetilde{a}_{i j} \widetilde{b}_{j} x_{j}=0\right.$ and $\left.\widetilde{a}_{i j} Z_{j} y_{j i}=0\right)=\mathbb{P}^{j}$, then $\mathbb{P}^{j}$ will have the following different expressions depend on the values of $x_{j}$ and $Z_{j} y_{j i}$,

$\mathbb{P}^{j}= \begin{cases}1, & \text { if } x_{j}=0, Z_{j} y_{j i}=0 ; \\ 1-p_{i j} q_{j}, & \text { if } x_{j}=1, Z_{j} y_{j i}=0 ; \\ 1-p_{i j}, & \text { if } x_{j}=0, Z_{j} y_{j i}=1 ; \\ 1-p_{i j}, & \text { if } x_{j}=1, Z_{j} y_{j i}=1 .\end{cases}$

When $x_{j}=0$ and $Z_{j} y_{j i}=0$, it is obvious that $\mathbb{P}^{j}=1$. When $x_{j}=1$, and $Z_{j} y_{j i}=0, \mathbb{P}^{j}=\mathbb{P}\left(\widetilde{a}_{i j} \widetilde{b}_{j}=0\right)$. Since the product of two random Bernoulli variables also follows a Bernoulli distribution and $\widetilde{a}_{i j} \sim \operatorname{Bernoulli}\left(p_{i j}\right), \widetilde{b}_{j} \sim$ Bernoulli $\left(q_{j}\right)$, then $\widetilde{a}_{i j} \widetilde{b}_{j} \sim$ Bernoulli $\left(p_{i j} q_{j}\right)$. Thus, $\mathbb{P}^{j}=\mathbb{P}\left(\widetilde{a}_{i j} \widetilde{b}_{j}=0\right)=1-$ $p_{i j} q_{j}$. When $x_{j}=0$ and $Z_{j} y_{j i}=1, \mathbb{P}^{j}=\mathbb{P}\left(\widetilde{a}_{i j}=0\right)=1-p_{i j}$. When $x_{j}=$ 1 and $Z_{j} y_{j i}=1, \mathbb{P}^{j}=\mathbb{P}\left(\widetilde{a}_{i j} \widetilde{b}_{j}=0\right.$ and $\left.\widetilde{a}_{i j}=0\right)=\mathbb{P}\left(\widetilde{a}_{i j}=0\right)$ (since $\widetilde{a}_{i j}=$ 0 is a subset of $\left.\widetilde{a}_{i j} \widetilde{b}_{j}=0\right)=1-p_{i j}$. To generalize the above four cases of $\mathbb{P}^{j}$, we can have $\mathbb{P}^{j}=\left(1-p_{i j} q_{j}\right)^{x_{j}\left(1-Z_{j} y_{j i}\right)}\left(1-p_{i j}\right)^{Z_{j} y_{j i}}$. The inequality (10e) follows by taking logarithm of both sides, while the inequality (10f) follows because $\log \varepsilon_{i} \in(-\infty, 0)$.

Considering both $Z_{i} y_{i i}=1$ and $Z_{i} y_{i i}=0$ under the case of $x_{i}=1$, we can get $Z_{i} y_{i i}+\sum_{j \in N\left(v_{i}\right)} \frac{\log \left(1-p_{i j} q_{j}\right)}{\log \varepsilon_{i}} x_{j}\left(1-Z_{j} y_{j i}\right)+\sum_{j \in N\left(v_{i}\right)} \frac{\log \left(1-p_{i j}\right)}{\log \varepsilon_{i}} Z_{j} y_{j i}+\frac{\log \left(1-q_{i}\right)}{\log \varepsilon_{i}} \geq 1$, $\forall v_{i} \in D$.

- $v_{i} \notin D, x_{i}=0$, the constraint (9) becomes

$$
\mathbb{P}\left(\sum_{j \in N\left(v_{i}\right)} \widetilde{a}_{i j} \widetilde{b}_{j} x_{j}+Z_{i} y_{i i}+\sum_{j \in N\left(v_{i}\right)} \widetilde{a}_{i j} Z_{j} y_{j i} \geq 1\right) \geq 1-\varepsilon_{i}, \forall v_{i} \notin D .
$$

, and two cases are shown as follows,

- $Z_{i} y_{i i}=0$, the inequality (11) becomes

$$
\sum_{j \in N\left(v_{i}\right)} \frac{\log \left(1-p_{i j} q_{j}\right)}{\log \varepsilon_{i}} x_{j}\left(1-Z_{j} y_{j i}\right)+\sum_{j \in N\left(v_{i}\right)} \frac{\log \left(1-p_{i j}\right)}{\log \varepsilon_{i}} Z_{j} y_{j i} \geq 1, \forall v_{i} \notin D .
$$

- $Z_{i} y_{i i}=1, \mathbb{P}\left(\sum_{j \in N\left(v_{i}\right)} \widetilde{a}_{i j} \widetilde{b}_{j} x_{j}+1+\sum_{j \in N\left(v_{i}\right)} \widetilde{a}_{i j} Z_{j} y_{j i} \geq 1\right) \geq 1-\varepsilon_{i}$ is always satisfied.

To sum up both $Z_{i} y_{i i}=0$ and $Z_{i} y_{i i}=1$ under the case of $x_{i}=0$, we have

$$
Z_{i} y_{i i}+\sum_{j \in N\left(v_{i}\right)} \frac{\log \left(1-p_{i j} q_{j}\right)}{\log \varepsilon_{i}} x_{j}\left(1-Z_{j} y_{j i}\right)+\sum_{j \in N\left(v_{i}\right)} \frac{\log \left(1-p_{i j}\right)}{\log \varepsilon_{i}} Z_{j} y_{j i} \geq 1, \forall v_{i} \notin D .
$$

Take both $v_{i} \in D$ and $i \notin D$ into consideration, we can reformulate (2) to the inequality under the contingencies from both line outages and PMU losses as,

$Z_{i} y_{i i}+\sum_{j \in N\left(v_{i}\right)} \frac{\log \left(1-p_{i j} q_{j}\right)}{\log \varepsilon_{i}} x_{j}\left(1-Z_{j} y_{j i}\right)+\sum_{j \in N\left(v_{i}\right)} \frac{\log \left(1-p_{i j}\right)}{\log \varepsilon_{i}} Z_{j} y_{j i}+\frac{\log \left(1-q_{i}\right)}{\log \varepsilon_{i}} x_{i} \geq 1$, 
$\forall v_{i} \in V$

As we did in the last subsection, $w_{i j}=x_{j} y_{j i}$ is also introduced to linearize the nonlinear term $x_{j} y_{j i}$ in (8).

\section{The Reliable Connected Power Dominating Set Problem}

A connected dominating set (CDS) is a dominating set with the property that each node in the subset can reach any other node in the subset by a path that stays entirely within this subset. The CDS problem is widely studied in wireless sensor networks $[20,21]$. Similarly, a connected power dominating set (CPDS) is a power dominating set $D$ in a power system with the property that each bus in $D$ can reach any other bus in $D$ by a path that stays entirely within $D$. Following the definition of CDS reliability proposed in [7], we propose the following reliable connected power dominating set (RCPDS) problem.

Assume $\widetilde{a}_{i j} \sim \operatorname{Bernoulli}\left(p_{i j}\right)$ and let $D$ be a CPDS of graph $G=(V, E)$. For a path $P$ between two distinct nodes $s, t \in D$, the delivery ratio of $(s, t)$ on this path is $D R_{P}(s, t)=\Pi_{(i, j) \in P} p_{i j}$, where $(i, j)$ is an edge in $P$. The delivery ratio of $(s, t)$ $\left(D R^{*}(s, t)\right)$ in a graph is the maximum delivery ratio among all paths, $D R^{*}(s, t)=$ $\max \left\{D R_{P}(s, t), \forall P\right.$ between $s$ and $\left.t\right\}$. Finally, the CPDS reliability $\left(R_{D}^{*}\right)$ is $R_{D}^{*}=$ $\min \left\{D R^{*}(s, t), \forall(s, t) \in D, s \neq t\right\}$. The RCPDS problem is to find a minimum CPDS $D$ satisfying $R_{D}^{*} \geq \sigma$, where $\sigma \in(0,1]$ denotes the reliability level of the PMU subgraph connectivity.

To solve this problem, we have the following theorem.

Theorem 3 Given $p_{i j}$ as the survival probability for each transmission line $(i, j)$, the constraint $R_{D}^{*} \geq \sigma$ is equivalent to ensure that the shortest paths among all $(s, t)$ pairs are less than or equal to $-\log \sigma$, where the length for each $(i, j) i s-\log \left(p_{i j}\right)$.

Proof. The requirement of reliability is $R_{D}^{*} \geq \sigma$, which is equivalent to $D R^{*}(s, t) \geq \sigma$ for each pair of $(s, t)$. Then, for any $(s, t)$ pair, the requirements $\max D R_{P}(s, t) \geq \sigma$ among all paths between $s$ and $t$ are equivalent to $\min -D R_{P}(s, t)=\min -\Pi_{(i, j) \in P} p_{i j} \leq$ $-\sigma$. With logarithm of both sides, we have $\min -\sum_{(i, j) \in P} \log \left(p_{i j}\right) \leq-\log \sigma$.

Given each edge $(i, j)$ as weight $-\log \left(p_{i j}\right)(>0)$, the problem becomes to find the shortest path among all the paths for each $(s, t)$ pair, which is $\min -\sum_{(i, j) \in P} \log \left(p_{i j}\right)$, and it should be no more than $-\log \sigma$.

Remark. It is worth mentioning that the shortest path between each pair of nodes less than or equal to $-\log (\sigma)$ is equivalent to the diameter $d$ of the graph $\leq-\log (\sigma)$.

In order to get a CPDS, we use the Miller-Tucker-Zemlin (MTZ) formulation proposed in [17]. A directed graph $G_{d}=(V \cup\{n+1, n+2\}, A)$ based on $G=(V, E)$ is constructed, where $A=\{(n+1, n+2)\} \cup\left\{\cup_{i=1}^{n}\{(n+1, i),(n+2, i)\}\right\} \cup E \cup E^{\prime}$, by introducing two additional nodes $n+1$ and $n+2$, adding arcs $(n+1, n+2),(n+1, i)$, $(n+2, i)$ for every $i$ in the original graph, and making each edge $(i, j)$ bi-directional. 
Table 1 Algorithm for minimum RCPDS

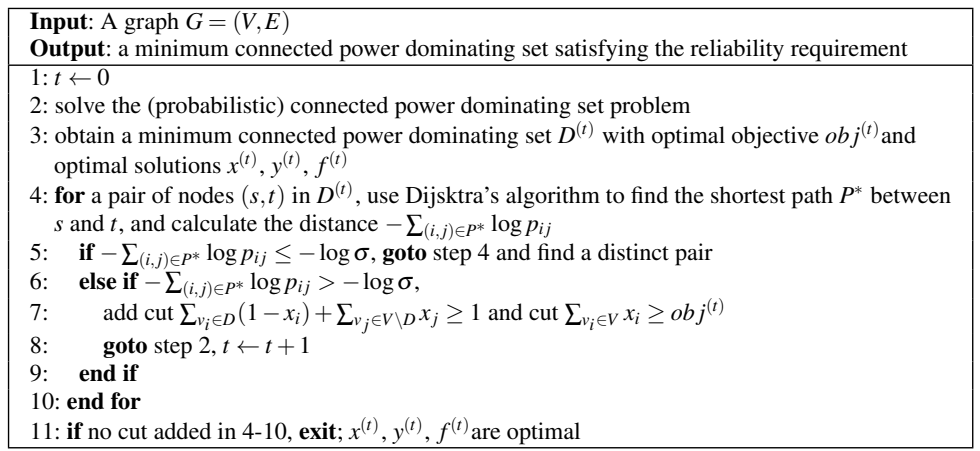

The MTZ formulation to ensure subgraph connectivity is presented as,

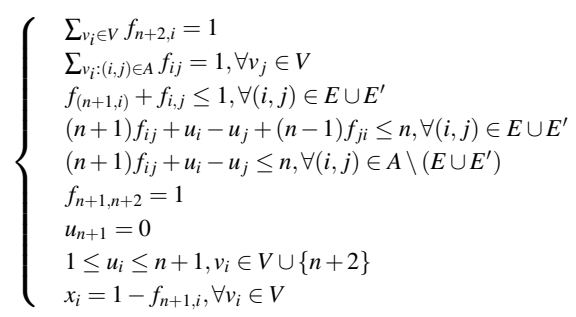

where $f_{i, j} \in\{0,1\}$ is denoted as whether the arc $(i, j)$ is selected to form a connected PDS or not. By this formulation, a directed spanning tree $T_{d}=\left(V \cup\{n+1, n+2\}, E_{d}\right)$ of $G_{d}$ is found by using $n+2$ to connect all the nodes inside the power dominating set and using $n+1$ as a root to connect $n+2$ and all the nodes not in the power dominating set. We refer [17] for more details.

Our algorithm to find the minimum CPDS to satisfy the reliability threshold $\sigma$ is shown in Table 1. In the algorithm, at each iteration $t$, we find a minimum CPDS $D^{(t)}$ and check if this $D^{(t)}$ satisfy the reliability requirement or not by checking whether the shortest paths of all the node pairs inside $D^{(t)}$ are less than or equal to $-\log \sigma$ or not. If it meets the requirement, we can claim $D^{(t)}$ is the optimal solution; if not, cuts $\sum_{v_{i} \in D}\left(1-x_{i}\right)+\sum_{v_{j} \in V \backslash D} x_{j} \geq 1$ and $\sum_{v_{i} \in V} x_{i} \geq o b j^{(t)}$ are added to the problem for further searching.

If random failures of PMUs are taken into account, the the delivery ratio of a node pair on a path can be similar. For a path $P$ between pair $s$ and $t$ consisting some nodes $v_{i}$ 's (with probability $q_{i}$ to survive) and edges $(i, j)$ 's (with probability $p_{i j}$ to survive), the delivery ratio of $(s, t)$ on this path is $D R_{P}(s, t)=\Pi_{i \in P,(i, j) \in P} q_{i} p_{i j}$.

The node splitting technique [22] can be applied to this problem in order to use Dijisktra's algorithm again. In each iteration, after obtaining the current minimum connected power dominating set $D^{(t)}$ with cardinality $\left|D^{(t)}\right|$ and before searching for the shortest paths between $(s, t)$ 's in a subgraph, constructing the subgraph is the main difference from previous subsection. When random failures come from only transmission lines, the shortest paths are searched inside the current minimum CPDS. 
However, when random failures come from both transmission lines and PMUs, the shortest paths are searched inside a subgraph with $\left|2 D^{(t)}\right|$ nodes. Assume that in iteration $t$, the minimum CPDS consists of buses $v_{i}$ 's. Shortest paths are searched on the directed graph $D^{(t)^{\prime}}$. To construct graph $D^{(t)^{\prime}}$, nodes $v_{i}{ }^{\prime}$ 's are introduced. For each node $v_{i}{ }^{\prime}$, there is an arc $\left(v_{i}{ }^{\prime}, v_{i}\right)$ with probability $q_{i}$ (the survival probability of bus $v_{i}$ in the original graph) to survive. For each node $v_{i}$, in the subgraph $D^{(t)}$, if there is an edge between $v_{i}$ and $v_{j}$ in the current minimum CPDS, then there is an arc from $v_{i}$ to $v_{j}^{\prime}$ in $D^{(t)^{\prime}}$ with probability $p_{i j}$. Then our goal becomes to check the shortest path from each $v_{i}^{\prime}$ to $v_{j}$ in $D^{(t)^{\prime}}$.

\section{Numerical Experiments}

The proposed IP formulations, their reformulations and the algorithm were all implemented in C++ and using CPLEX 12.3 via ILOG Concert Technology 2.9, and all computations were performed on a Linux machine with 4 Intel(R) Xeon(TM) CPU $3.60 \mathrm{GHz}$ processors and 32GB RAM. Computational time is reported in CPU seconds.

In Fig. 2, we perform computation on 6 IEEE test cases (IEEE 14-Bus, IEEE 30Bus, IEEE 57-Bus, RTS-96, IEEE 118-Bus, and IEEE 300-Bus, see [23]) with different given reliability levels $\varepsilon$ 's and survival probabilities of transmission lines $p_{i j}$ 's. The optimal objective values are shown in the figure. In Fig. 3, the PMU failures with survival probability $q_{i}$ 's are also considered under the reliability level of $\varepsilon_{i}=0.1$. The results are quite reasonable that given the same value of $\varepsilon$, the optimal objective value (the minimum required number of PMUs) becomes smaller when the probability $p_{i j}$ or the probability $q_{i}$ increases. It means that when the transmission lines and the PMUs have higher chances to survive, the amount of necessary installed PMUs is getting lower. On the other hand, when the probability $p_{i j}$ or $q_{i}$ remains the same, the optimal objective value increases when the value $\varepsilon$ decreases. This also makes sense since the requirement for each bus to be observed is getting stricter when $\varepsilon$ is becoming lower.

The results for RCPDS problem based on 6 test cases are shown in Table 3 and 4. During the experiments, most transmission lines and PMUs work well and have high chance to survive $(0.98)$ as in real world, while some of them (about $5 \%$ of the total amount) have probability 0.5 to fail. The lines and PMUs with high failing probabilities are randomly selected as shown in Table 2 .

In Table 5, the results of different models are compared and shown. Models PPDS, RCPDS and PPDS+RCPDS use the same line probabilities as we did for tests in Table 3, that is most of the transmission lines are stable with survival probability 0.98 , and some of lines have high probabilities 0.5 to fail as shown in Table 2 . For the models RCPDS and PPDS+RCPDS, we test them with transmission line failures only, since with current data, some of the results would turn out to be infeasible with both transmission line and PMU failures. RCPDS and PPDS+RCPDS are tested based on the same thresholds $\sigma$ 's, which are $0.490,0.479,0.380,0.501,0.200$ and 0.01 for IEEE 14-Bus, IEEE 30-Bus, IEEE 57-Bus, RTS-96, IEEE 118-Bus and IEEE 300-Bus systems respectively. For models PPDS and PPDS+RCPDS, 0.02 is 
Table 2 Selected lines/ PMUs with high failure probability for RCPDS

\begin{tabular}{|c|c|c|c|c|}
\hline & \# failed PMUs & \# failed lines & failed PMUs & failed lines \\
\hline $\begin{array}{l}\text { IEEE 14- } \\
\text { Bus }\end{array}$ & 1 & 1 & 8 & $(5,6)$ \\
\hline $\begin{array}{l}\text { IEEE 30- } \\
\text { Bus }\end{array}$ & 2 & 2 & 3,25 & $(6,8),(27,28)$ \\
\hline $\begin{array}{l}\text { IEEE 57- } \\
\text { Bus }\end{array}$ & 3 & 4 & $11,12,40$ & $(4,18),(12,17),(20,21),(41,56)$ \\
\hline RTS-96 & 4 & 5 & $9,32,58,72$ & $(8,10),(17,18),(30,34),(68,71)$ \\
\hline $\begin{array}{l}\text { IEEE } \\
118 \text {-Bus }\end{array}$ & 6 & 9 & $\begin{array}{l}7,15,40,47,88, \\
102\end{array}$ & $\begin{array}{l}(5,11),(15,17),(23,32),(45,46),(54, \\
56),(56,59), \quad(92,100),(110,112), \\
(75,118)\end{array}$ \\
\hline $\begin{array}{l}\text { IEEE } \\
\text { 300-Bus }\end{array}$ & 15 & 20 & $\begin{array}{l}1,21,41,61,81, \\
101,121,141,161, \\
181,201,221,241, \\
261,281\end{array}$ & $\begin{array}{l}(31,266),(270,296),(271,272), \\
(294,300),(1,5),(4,16),(13,19), \\
(18,20),(23,231),(25,232),(40,68), \\
(49,50),(52,54),(60,64),(70,71), \\
(84,86),(245,99),(102,104),(105, \\
106),(122,124)\end{array}$ \\
\hline
\end{tabular}

Table 3 RCPDS with failures of only transmission lines

\begin{tabular}{|c|c|c|c|c|}
\hline & $\sigma$ & time & obj & placement \\
\hline \multirow{2}{*}{ IEEE 14-Bus } & 0.479 & 0.01 & 4 & $4,5,6,9$ \\
\hline & 0.490 & 0.24 & 6 & $2,4,6,9,10,11$ \\
\hline \multirow{3}{*}{ IEEE 30-Bus } & 0.447 & 0.09 & 9 & $2,4,6,10,12,15,18,27,28$ \\
\hline & 0.457 & 0.13 & 9 & $2,4,6,10,12,15,20,27,28$ \\
\hline & 0.851 & 7.95 & 11 & $2,4,6,10,12,15,18,23,24,25,27$ \\
\hline \multirow[t]{2}{*}{ IEEE 57-Bus } & 0.407 & 10.45 & 24 & $\begin{array}{l}1,6,7,8,9,10,11,13,15,20,21,22,23,24,25,29,30,31,32, \\
38,41,49,52,53\end{array}$ \\
\hline & 0.417 & 19.25 & 24 & $\begin{array}{l}1,6,7,8,9,10,11,13,15,20,21,22,23,24,25,29,30,31,32, \\
38,41,49,54,55\end{array}$ \\
\hline \multirow[t]{2}{*}{ RTS-96 } & 0.708 & $>3000$ & 28 & $\begin{array}{l}2,6,10,11,13,14,15,16,21,25,26,27,32,33,39,40,48,50, \\
54,56,57,58,59,61,62,64,71,73\end{array}$ \\
\hline & 0.721 & $>3000$ & 28 & $\begin{array}{l}2,6,10,11,13,14,15,16,21,25,26,27,32,33,39,40,48,53, \\
54,56,57,58,59,61,62,64,71,73\end{array}$ \\
\hline \multirow[t]{2}{*}{ IEEE 118-Bus } & 0.347 & 40.85 & 39 & $\begin{array}{l}3,5,8,12,14,15,19,20,23,24,27,28,32,34,37,40,42,45, \\
49,50,51,54,62,66,69,70,75,77,80,85,86,89,92,94,99, \\
100,103,105,110\end{array}$ \\
\hline & 0.355 & 48.35 & 39 & $\begin{array}{l}3,5,8,12,14,15,19,22,23,24,27,31,32,34,37,40,42,45, \\
49,50,51,54,59,66,69,70,75,77,80,85,86,89,92,94,98, \\
100,103,105,110\end{array}$ \\
\hline \multirow[t]{2}{*}{ IEEE 300-Bus } & 0.126 & $>3000$ & 112 & $\begin{array}{l}1,2,3,8,11,14,15,17,18,20,22,23,24,25,31,32,37,38,41, \\
43,47,48,49,53,54,55,56,58,59,60,61,64,66,79,81,83, \\
84,85,88,89,92,93,94,98,99,100,101,105,106,107,112, \\
113,116,118,119,121,122,123,132,134,135,136,140,141, \\
142,143,154,157,158,160,161,163,169,175,177,179,185, \\
186,189,190,191,193,194,195,196,198,199,203,204,205, \\
208,210,211,213,216,217,221,224,227,228,266,267,268, \\
269,270,271,272,273,274,276,291,294\end{array}$ \\
\hline & 0.129 & $>3000$ & 120 & $\begin{array}{l}1,2,3,8,11,14,15,17,18,20,22,23,24,25,31,32,37,38, \\
39,43,47,48,49,53,54,55,56,58,59,60,61,62,64,74,76, \\
79,81,83,84,85,88,89,93,94,98,99,100,101,104,105,106, \\
108,109,113,116,118,119,121,122,123,124,129,132,134, \\
135,140,141,142,143,154,155,159,161,163,167,169,172, \\
174,175,177,179,183,184,185,186,189,190,191,193,194, \\
198,199,203,204,205,207,208,210,211,213,214,216,217, \\
221,224,226,227,228,266,267,268,269,270,271,272,273, \\
274,276,291,294\end{array}$ \\
\hline
\end{tabular}




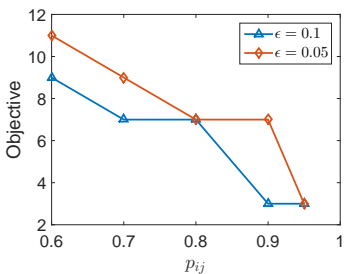

(a) IEEE 14-Bus

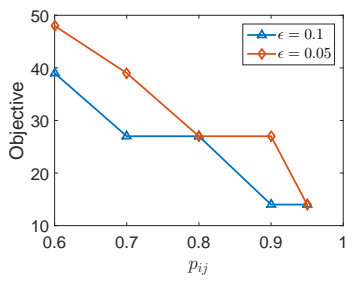

(d) RTS-96

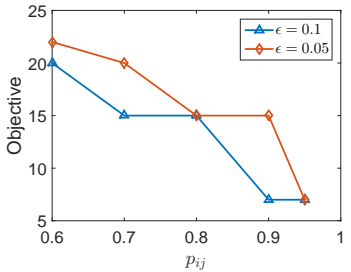

(b) IEEE 30-Bus

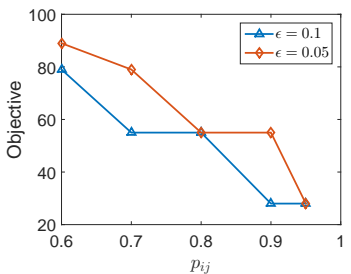

(e) IEEE 118-Bus

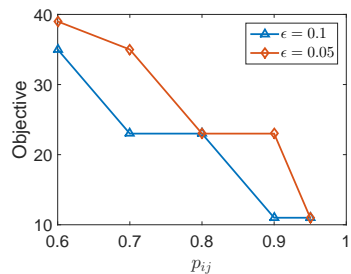

(c) IEEE 57-Bus

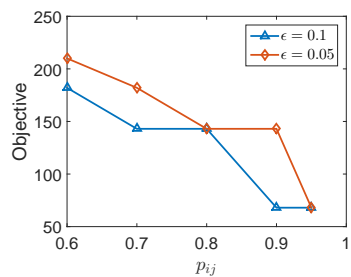

(f) IEEE 300-Bus

Fig. 2 PPDS with failures of only transmission lines

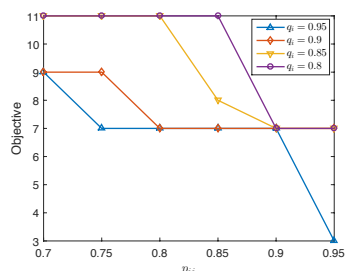

(a) IEEE 14-Bus

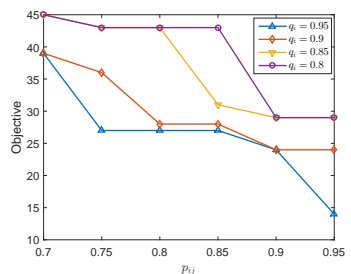

(d) RTS-96

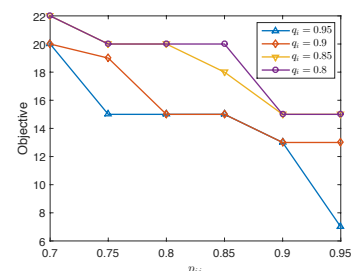

(b) IEEE 30-Bus

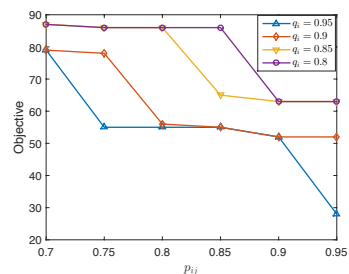

(e) IEEE 118-Bus

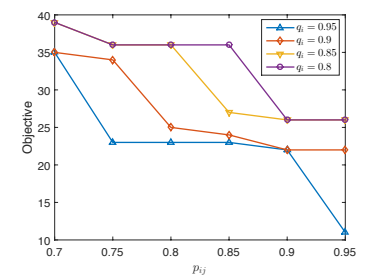

(c) IEEE 57-Bus

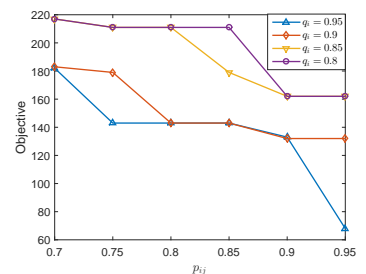

(f) IEEE 300-Bus

Fig. 3 PPDS with failures of both transmission lines and PMUs

selected as $\varepsilon$ 's to make reliability level as 0.98 . From this table, under the same contingency situation, PPDS+RCPDS needs most number of PMUs to be installed since it needs to meet the requirements come from two parts, including the reliability of covering each bus in the system and the reliability for the resulted subgraph to be connected. In general, the objective (the needed PMUs to be installed) for each system reduces in the order of PPDS+RCPDS, RCPDS, CPDS, PPDS and PDS, which is basically the reducing order of requirements. 
Table 4 RCPDS with failures of both transmission lines and PMUs

\begin{tabular}{|c|c|c|c|c|}
\hline & $\sigma$ & time & obj & placement \\
\hline \multirow{2}{*}{ IEEE 14-Bus } & 0.437 & 0.01 & 4 & $4,5,6,9$ \\
\hline & 0.447 & 0.32 & 6 & $2,4,6,9,10,11$ \\
\hline \multirow{2}{*}{ IEEE 30-Bus } & 0.390 & 0.1 & 9 & $2,4,6,10,12,15,18,27,28$ \\
\hline & 0.407 & 0.09 & 9 & $2,4,6,10,12,15,20,27,28$ \\
\hline \multirow[t]{2}{*}{ IEEE 57-Bus } & 0.191 & 10.49 & 24 & $\begin{array}{l}1,6,7,8,9,10,11,13,15,20,21,22,23,24,25,29,30,31,32 \\
38,41,49,52,53\end{array}$ \\
\hline & 0.195 & 18.02 & 24 & $\begin{array}{l}3,4,7,8,9,11,12,13,15,18,22,23,24,25,29,30,31,32,38 \\
41,49,50,52,55\end{array}$ \\
\hline \multirow[t]{2}{*}{ RTS-96 } & 0.251 & $>3000$ & 28 & $\begin{array}{l}2,6,10,11,13,14,15,16,21,25,26,27,32,33,39,40,48,50 \\
54,56,57,58,59,61,62,64,71,73\end{array}$ \\
\hline & 0.257 & $>3000$ & 28 & $\begin{array}{l}1,2,5,10,11,13,14,16,25,27,32,33,34,36,39,40,47,48 \\
49,50,52,56,57,59,62,64,65,66\end{array}$ \\
\hline \multirow[t]{2}{*}{ IEEE 118-Bus } & 0.123 & 40.86 & 39 & $\begin{array}{l}3,5,8,12,14,15,19,20,23,24,27,28,32,34,37,40,42,45 \\
49,50,51,54,62,66,69,70,75,77,80,85,86,89,92,94,99 \\
100,103,105,110\end{array}$ \\
\hline & 0.126 & 41.36 & 39 & $\begin{array}{l}3,5,8,12,14,15,19,20,23,24,27,31,32,34,37,40,42,45 \\
49,51,54,56,62,66,69,70,75,77,80,85,86,89,92,94,96 \\
100,103,105,110\end{array}$ \\
\hline \multirow[t]{2}{*}{ IEEE 300-Bus } & 0.0051 & $>3000$ & 112 & $\begin{array}{l}1,2,3,8,11,14,15,17,18,20,22,23,24,25,31,32,37,38,41, \\
43,47,48,49,53,54,55,56,58,59,60,61,64,66,79,81,83, \\
84,85,88,89,92,93,94,98,99,100,101,105,106,107,112, \\
113,116,118,119,121,122,123,132,134,135,136,140,141, \\
142,143,154,157,158,160,161,163,169,175,177,179,185, \\
186,189,190,191,193,194,195,196,198,199,203,204,205, \\
208,210,211,213,216,217,221,224,227,228,266,267,268, \\
269,270,271,272,273,274,276,291,294\end{array}$ \\
\hline & 0.0052 & $>3000$ & 115 & $\begin{array}{l}1,2,3,8,11,14,15,17,18,20,22,23,24,25,31,32,37,38,43, \\
47,48,49,53,54,55,56,58,59,60,64,67,74,76,79,81,83, \\
84,85,88,89,93,94,98,99,100,101,104,105,106,107,112, \\
113,116,118,119,121,122,123,124,132,134,135,140,141, \\
142,143,154,155,158,160,161,163,169,172,174,175,177 \\
179,184,185,186,189,190,191,193,196,198,199,203,204, \\
205,207,208,210,211,213,216,217,221,224,226,227,228, \\
266,267,268,269,270,271,272,273,274,276,291,294\end{array}$ \\
\hline
\end{tabular}

Table 5 Comparison of objectives with different models

\begin{tabular}{|l|c|c|c|c|c|}
\hline & PDS & CPDS & PPDS & RCPDS & PPDS\&RCPDS \\
\hline IEEE 14-Bus & 3 & 4 & 3 & 6 & 6 \\
IEEE 30-Bus & 7 & 9 & 7 & 9 & 11 \\
IEEE 57-Bus & 11 & 24 & 11 & 24 & 26 \\
RTS-96 & 14 & 28 & 14 & 28 & 29 \\
IEEE 118-Bus & 28 & 39 & 31 & 39 & 41 \\
IEEE 300-Bus & 68 & 112 & 72 & 112 & 120 \\
\hline
\end{tabular}

\section{Conclusions}

In this paper, with consideration of random failures of transmission lines or PMUs in a power system, we present two problems: one considering reliability of coverage of each bus, the other considering reliability of connectivity of the PMU subgraph. In order to solve the IP formulations of these problems, some reformulation techniques are applied and some algorithms are designed. Additionally, the two problems can be combined to meet the reliability requirement of both coverage and connectivity sides. For some similar combinatorial optimization problems, such as sensor placement and coloring problems, the proposed approaches can be generalized to consider the uncertainty of edges in a random graph. 
Acknowledgement. We would appreciate the initial discussion with Dr. Yiwen $\mathrm{Xu}$ on this research.

\section{References}

1. Haynes, T. W., Hedetniemi, S. M., Hedetniemi, S. T., \& Henning, M. A.: Domination in graphs applied to electric power networks. SIAM Journal on Discrete Mathematics. 15 (4), 519-529 (2002)

2. Garey, M. R., Johnson, D. S.: Computers and intractability: A guide to the theory of NP-completeness. Bulletin (New Series) of the American Mathematical Society. 3 (2), 898-904 (1980)

3. Dua, D., Dambhare, S., Gajbhiye, R. K., \& Soman, S. A.: Optimal multistage scheduling of PMU placement. An ILP approach. IEEE Transactions on Power Delivery. 23 (4), 1812-1820 (2008)

4. Sun, O., Fan, N.: Solving the multistage PMU placement problem by integer programming and equivalent network design model. Journal of Global Optimization, DOI: 10.1007/s10898-018-0672-8 (2018).

5. Fan, N., Watson, J.: On integer programming models for the multi-channel PMU placement problem and their solution. Energy Systems. 6 (1), 1-19 (2015)

6. Qiu, F.: Probabilistic covering problems. Diss. Georgia Institute of Technology. (2013)

7. He, J. S., Cai, Z., Ji, S., Beyah, R., \& Pan, Y: A genetic algorithm for constructing a reliable MCDS in probabilistic wireless networks. International Conference on Wireless Algorithms, Systems, and Applications. 96-107 (2011)

8. Boria, N., Murat, C., \& Paschos, V. T.: The probabilistic minimum dominating set problem. Discrete Applied Mathematics. (2016)

9. Mazhari, S. M., Monsef, H., Lesani, H., \& Fereidunian, A.: A multi-objective PMU placement method considering measurement redundancy and observability value under contingencies. IEEE Transactions on Power Systems. 28 (3), 2136-2146 (2013)

10. Aghaei, J., Baharvandi, A., Rabiee, A., \& Akbari, M. A.: Probabilistic PMU placement in electric power networks: an MILP-based multiobjective model. IEEE Transactions on Industrial Informatics. 11 (2), 332-341 (2015)

11. Abbasy, N. H., \& Ismail, H. M.: A unified approach for the optimal PMU location for power system state estimation. IEEE Transactions on Power Systems. 24 (2),806-813 (2009)

12. Chakrabarti, S., Kyriakides, E., \& Eliades, D. G.: Placement of synchronized measurements for power system observability. IEEE Transactions on Power Delivery. 24 (1), 12-19 (2009)

13. Aminifar, F., Khodaei, A., Fotuhi-Firuzabad, M., \& Shahidehpour, M.: Contingency-constrained PMU placement in power networks. IEEE Transactions on Power Systems. 25 (1), 516-523 (2010)

14. Abiri, E., Rashidi, F., \& Niknam, T.: An optimal PMU placement method for power system observability under various contingencies. International Transactions on Electrical Energy Systems. 25 (4), 589-606, (2015)

15. Aminifar, F., Fotuhi-Firuzabad, M., Shahidehpour, M., \& Khodaei, A.: Probabilistic multistage PMU placement in electric power systems. IEEE Transactions on Power Delivery. 26 (2), 841-849 (2011)

16. Gomez, O., Rios, M. A., \& Anders, G.: Reliability-based phasor measurement unit placement in power systems considering transmission line outages and channel limits. IET Generation, Transmission \& Distribution. 8 (1), 121-130 (2014)

17. Fan, N., Watson, J.P.: Solving the connected dominating set problem and power dominating set problem by integer programming. Combinatorial Optimization and Applications. 371-383 (2012)

18. Ahmed, S.: Convex relaxations of chance constrained optimization problems. Optimization Letters, $8(1), 1-12(2014)$

19. Haight, R. G., Charles, S. R., and Stephanie A. S.: An integer optimization approach to a probabilistic reserve site selection problem. Operations Research. 48(5), 697-708 (2000)

20. Ding, L., Gao, X., Wu, W., Lee, W., Zhu, X., \& Du, D. Z.: An exact algorithm for minimum CDS with shortest path constraint in wireless networks. Optimization Letters. 5(2), 297-306 (2011)

21. Verma, A., Butenko, S.: A distributed approximation algorithm for the bottleneck connected dominating set problem. Optimization Letters. 6(8), 1583-1595 (2012)

22. Ahuja, R. K., Magnanti, T.L., Orlin, J.B.: Network Flows: Theory, Algorithms, and Applications Prentice Hall, Englewood Cliffs. 41-42 (1993)

23. IEEE reliability test data (2012), http://www.ee.washington.edu/research/pstca/ 